\title{
THIN POSITION AND THE RECOGNITION PROBLEM FOR $\mathrm{S}^{3}$
}

\section{Abigail ThOMPSON}

\begin{abstract}
We describe a modified version of Rubinstein's algorithm to detect the 3-sphere and use thin position combined with standard 3-manifold techniques to prove that the algorithm works.
\end{abstract}

\section{Introduction}

In the spring of 1992, Hyam Rubinstein gave a series of lectures at the Technion, Haifa, describing an algorithm to determine whether or not a triangulated 3-manifold is the 3-sphere. Rubinstein's proof that his algorithm works uses the language of PL minimal surface theory. The substance of this paper is a different approach to the proof that the algorithm works, using techniques from knot theory. In addition, we simplify the original algorithm by using a modified definition of almost normal 2-sphere.

There are two interesting points. One is simply that an algorithm exists, requiring for its proof only fairly standard normal surface theory and knot theory. The second is that PL-minimal surface theory and knot theory are so closely connected; a given tool or concept in one area seems to have its counterpart in the other. The correspondence between the two is likely to be a fruitful area for further study.

Theorem 1. [8] There exists an algorithm to determine whether or not a compact 3-manifold is $S^{3}$.

We give some definitions, followed by an outline of the proof.

Definitions. Let $M$ be a closed orientable 3-manifold with fixed triangulation $T$. An arc in a 2-cell of $T$ is normal if it connects distinct edges of the 2-cell. Let $c$ be a simple closed curve on the boundary of a tetrahedron $H$ in the triangulation, such that $c$ intersects each face of $H$ in normal arcs. $c$ is a normal curve. The length of $c$ is the number of times $c$ crosses the edges of $H$. A closed orientable surface $F$ imbedded in $M$ is a normal surface if $F$ intersects each tetrahedron in $T$ in a collection

Received July 14, 1994.

The author was partially supported by the National Science Foundation and the Alfred P. Sloan Foundation. 


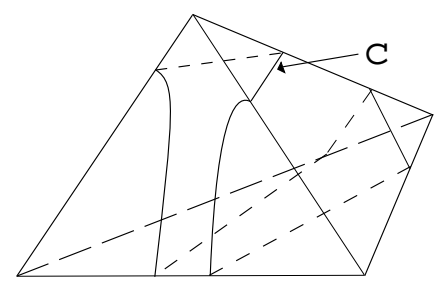

Figure 1. A normal curve of length eight.

of disks all of whose boundary curves are normal curves of length three or four, i.e., in normal triangles and quadrilaterals. $F$ is almost normal if $F$ intersects each tetrahedron in $T$ in a collection of normal triangles and quadrilaterals and, in one of the tetrahedra, precisely one disk whose boundary is a normal curve $c$ of length eight, and possibly some normal triangles.

Note. These definitions differ slightly from Rubinstein's; in particular, he considers a more general type of surface to be almost normal.

Outline of the proof of Theorem 1. Let $\Sigma$ be a maximal collection of disjoint non-parallel (in $(M, T)$ ) normal 2 -spheres in $M$. Notice that $\Sigma$ is not empty, since the boundary of a small neighborhood of each vertex is a normal 2-sphere in $M . \Sigma$ cuts $M$ into three types of components $M_{0}$ :

(1) $M_{0}$ is a 3 -ball containing a single vertex.

(2) $M_{0}$ has more than one boundary component.

(3) $M_{0}$ has exactly one boundary component and is not of type 1 .

Using simplicial homology, we can algorithmically check that $H_{1}\left(M ; Z_{2}\right)$ is trivial to ensure that $M$ contains no closed non-orientable or nonseparating surfaces. In particular we assume from now on that all 2-spheres are separating.

Notice that components of type 2 and 3 contain no vertices of $T$. We prove the following lemmas:

Lemma 2. A component of type 2 is a punctured 3-ball.

Lemma 4. A component of type 3 is a 3-ball if and only if it contains an almost normal 2-sphere.

Assuming lemmas 2 and 4, the algorithm is as follows:

(1) Search for $\Sigma$, a maximal collection of disjoint, non-parallel normal 2-spheres.

(2) Consider each component of $M-\Sigma$. In each component with a single 2-sphere boundary, search for an almost normal 2-sphere. 
(3) The manifold is $S^{3}$ if and only if each component considered in step 2 contains an almost normal 2-sphere.

The search for $\Sigma$ is accomplished via an algorithm due to Haken [3], [4], [6], [7]. Haken's work does not describe how to find almost normal 2 -spheres, but a modification of the algorithm suffices.

Outline of the paper: Section 2 will be devoted to the proof of lemma 2. Section 3 consists of an unknotting lemma, which is used in section 4 to prove lemma 4 . Section 5 will discuss the necessary modification of Haken's algorithm.

We first give some additional definitions, state some facts about normal curves on the boundary of a tetrahedron and prove two useful claims.

Definitions. A separating surface $F$ properly imbedded in a 3-manifold is strongly compressible if there exist disjoint compressing disks for $F$ lying on opposite sides of $F$. If $F$ is not strongly compressible it is weakly incompressible. Note that a separating surface incompressible to one or both sides is weakly incompressible.

Let $F$ be a closed orientable surface imbedded in a 3-manifold $M^{3}$. Let $\mathrm{X}$ be a 1-complex properly imbedded in $M$, possibly puncturing $F$. We define incompressible in the complement of $X$, strongly compressible in the complement of $X$ and weakly incompressible in the complement of $X$ in the obvious manner.

A parallel 2-sphere is a 2-sphere punctured twice by a single edge of $T$, boundary parallel to that edge.

Facts about normal curves on the boundary of a tetrahedron $H$ :

(1) A normal curve of odd length has length 3.

(2) There are no normal curves of length 6.

(3) Any normal curve of length greater than 8 crosses some edge of $H$ at least 3 times.

Proof. First flatten the 1-skeleton of the tetrahedron out in the plane. The proofs then proceed by first drawing in the vertices of the 1-skeleton, then drawing in the normal curve $c$, which, w.l.o.g., can be drawn as a round circle, and then adding in the edges of the 1-skeleton, which now may look quite complicated (the more painful details are left to the reader):

(1) Any normal curve $c$ of odd length must separate one vertex of $H$ from the other three; such a curve can only have length three.

(2) Any normal curve $c$ of even length separates the vertices of $H$ in pairs. Suppose it separates $v$ and $v^{\prime}$ from $w$ and $w^{\prime}$. Examine the disk $D \subset \partial \mathrm{H}$ bounded by $c$, containing $v$ and $v^{\prime}$. If an edge of $H$ lies entirely inside $D$, then $c$ has length 4 . Suppose no edge of $H$ lies entirely inside 

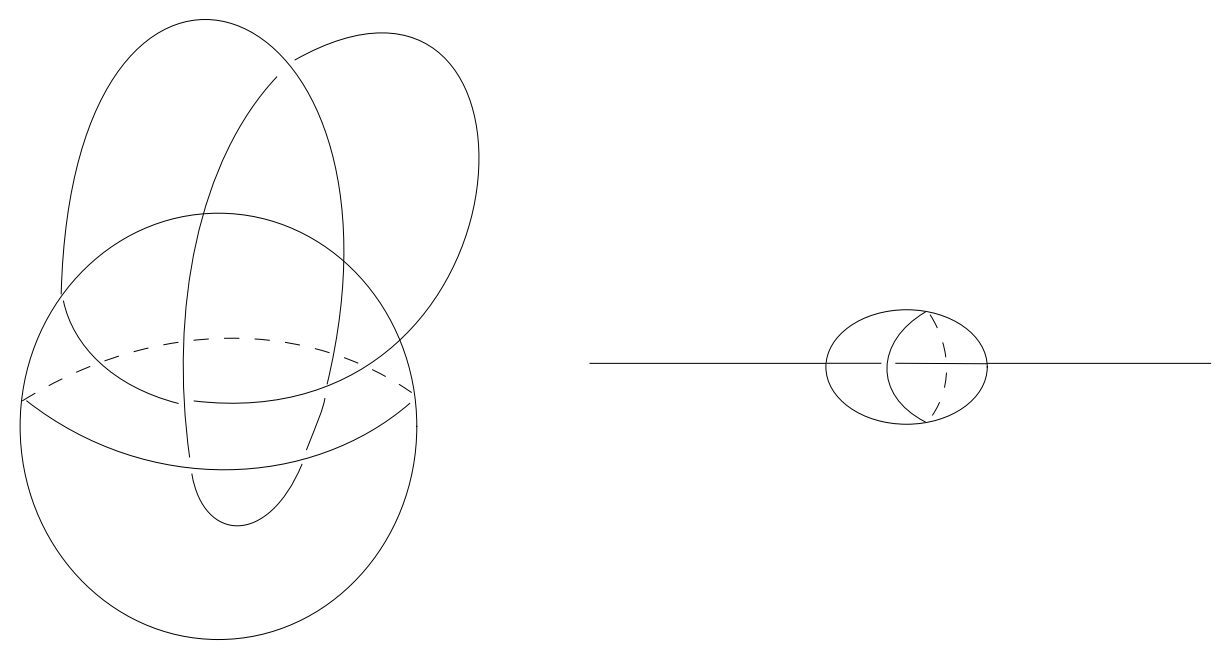

FiguRE 2. A 2-sphere in $S^{3}$, weakly incompressible in the complement of the trefoil, and a parallel 2-sphere.

$D$; by considering the complementary disk $D^{\prime}=\partial H-D$, one can see that the length of $c$ is at least 8 .

(3) Suppose $c$ is a normal curve of length greater than 8. Note that every edge of $H$ connecting a vertex in $D$ to a vertex in $D^{\prime}$ intersects $c$ an odd number of times, and the edges connecting $v$ to $v^{\prime}$ and $w$ to $w^{\prime}$ each intersect $c$ an even number of times. Counting suffices to show that some edge intersects $c$ at least three times.

The following claim says that normal surfaces in $(M, T)$ are roughly the same as surfaces which are incompressible in the complement of the 1-skeleton. More precisely:

Claim 1.1. A closed orientable normal surface $F$ in $(M, T)$ is incompressible in the complement of the 1-skeleton of $T$. If $F$ is a closed orientable surface which is incompressible in the complement of the 1-skeleton of $T$, there exists an ambient isotopy rel the 1-skeleton of $T$ from $F$ to a normal surface $\tilde{F}$ or to a parallel 2-sphere.

Proof. An innermost disk/outermost arc argument suffices for the first part of the claim. Now suppose $F$ is a closed orientable surface in $(M, T)$ which is incompressible in the complement of the 1-skeleton. Pick an innermost simple closed curve $c$ of intersection between $F$ and a face of one of the tetrahedra. The curve $c$ bounds a disk $D$ in the face with interior disjoint from $F$, and hence it must also bound a disk $D^{\prime}$ in $F$ disjoint from the 1skeleton. $D \cup D^{\prime}$ together bound a 3 -ball in $M$ disjoint from the 1-skeleton. 


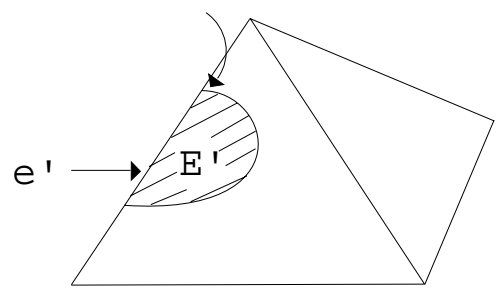

$\mathrm{H}$

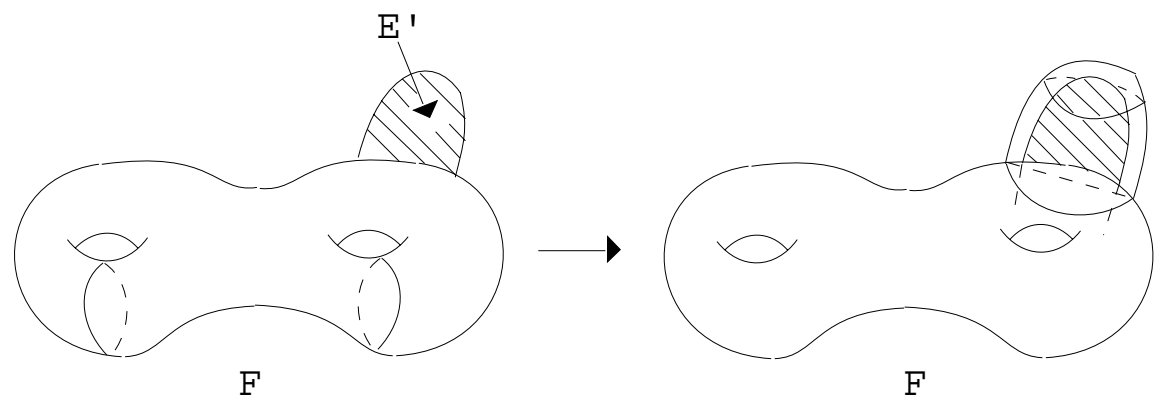

Figure 3. Doubling a boundary compression to yield a compressing disk.

Isotop $F$ across the 3-ball to remove the curve of intersection $c$. Hence we can assume that $F$ intersects any face of a given tetrahedron in arcs.

Let $E$ be some face of a tetrahedron. Suppose some arc $\beta$ of $F \cap E \subset E$ connects an edge of $E$ to itself. Assume $\beta$ is an outermost such arc in $E$. Then $\beta$ cuts off a subdisk $E^{\prime}$ of $E$, which one can think of as a boundary compressing disk for $F$ with respect to the 1-skeleton. One can construct a disk with boundary imbedded on $F$ in the complement of the 1-skeleton by banding together two copies of $E^{\prime}$ across $\partial E^{\prime}-\beta$.

We will call this operation doubling a boundary compression (see figure 3). Unless $F$ is a 2-sphere parallel to the edge of $E$ incident to $\alpha$, this operation produces a compressing disk for $F$ in the complement of $T_{0}$. Hence we can assume that every arc $\beta$ of $F \cap E \subset E$ connects distinct edges of $E$.

Let $H$ be any tetrahedron in $T$. Examine $F \cap H$. We need to show that if $F \cap H$ consists of anything but normal triangles and quadrilaterals, then $F$ is compressible in the complement of the 1-skeleton.

Suppose $F \cap H$ contains a component $\tilde{F}$ which is not a disk. Then $F \cap H$ is compressible in $H$. A compressing disk for $F \cap H$ in $H$ is a compressing disk for $F$ in the complement of the 1-skeleton (one needs to check such a disk is not trivial when regarded as a compressing disk for $F$ ), hence $F$ 


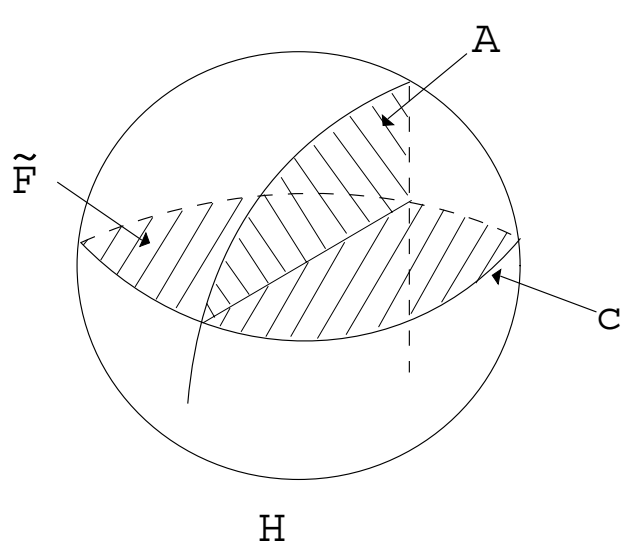

FigURE 4

is compressible in the complement of the 1-skeleton. So we can assume all components of $F \cap H$ are disks.

Using facts 1, 2 and 3 about normal curves on the boundary of $H$, as well as the observation that a normal curve of length eight on the boundary of $H$ must intersect some edge $e$ of $H$ at least twice, we see that if $F \cap H$ consists of anything but normal triangles and quadrilaterals, then there is some component $\tilde{F}$ of $F \cap H$ whose boundary is a normal curve $c$ intersecting some edge $E$ of $H$ at least twice. Then there exists an imbedded disk $A$, with $\partial A=\alpha \cup \beta$, where $\alpha \subset E$ and $\beta \subset \tilde{F}$ (see figure 4).

If the interior of $A$ is disjoint from $F$, we can either double $A$ across $\alpha$ to produce a compressing disk for $F$ in the complement of the 1-skeleton, or conclude that $F$ is a 2-sphere parallel to $\alpha$. If the interior of $A$ intersects $F$, we first do an outermost arc/innermost disk argument on $A \cap F \subset A$, and then reach the same conclusion.

Let $(S, \partial S)$ be a connected orientable separating surface properly imbedded in a 3-manifold $(M, \partial M) . S$ splits $M$ into two pieces; call these $M_{1}$ and $M_{2}$. Suppose $S$ is compressible into both $M_{1}$ and $M_{2}$. Let $\mathbf{D}_{i}, \quad i=1,2$, be a minimal complete collection of compressing disks for $S$ in $M_{i}$; each $\mathbf{D}_{i}$ is non-empty by hypothesis. Let $W^{\prime}$ be the 3-manifold obtained by attaching 2-handles to a small neighborhood $S \times I$ of $S$ along the collection $\mathbf{D}=\mathbf{D}_{1} \cup \mathbf{D}_{2}$. If any component of $\partial W^{\prime}$ bounds a 3-ball in $M$ disjoint from $W^{\prime}$, fill it in. Call the resulting object $W$. Then: 
Claim 1.2. At least one of the following must hold:

(1) $M-S$ is reducible (this includes the possibility that $M$ has a 2sphere boundary component disjoint from $S$ ).

(2) $M_{1}$ and $M_{2}$ are compression bodies, with $\partial_{+} M_{i}=S$.

(3) $S$ is strongly compressible in $M$.

(4) $\partial W$ is incompressible in $M$. Note that this implies that every component of $\partial W$ is incompressible in $M$. Hence either

(a) $M$ contains an incompressible non-boundary parallel surface $F . F$ is disjoint from $S$, and $F$ is obtained by compressing $S$ entirely to one side. or

(b) $\partial W$ consists of a collection of components, each of which is parallel to some component of $\partial M$.

Proof. This is an application of Casson-Gordon's work on Heegaard splittings (see Lemma 1.1 in [1]).

If $\partial W=\emptyset$, then (2) holds. Assume $\partial W \neq \emptyset$.

Suppose some component of $\partial W$ is a 2 -sphere. Then (1) holds.

Assume no component of $\partial W$ is a 2 -sphere. Then $W$ has a natural Heegaard splitting with splitting surface $S$.

Assume $\partial W$ is compressible in $M$. Since $\mathbf{D}_{1}$ and $\mathbf{D}_{2}$ are complete, $\partial W$ is incompressible in $M-W$. Hence $\partial W$ must be compressible in $W$. By [1], this implies that the Heegaard splitting of $w$ by $S$ is weakly reducible. Hence $S$ is strongly compressible in $M$, and (3) holds.

If $\partial W$ is incompressible in $M$, then each component of $\partial W$ is incompressible in $M$, and either (4a) or (4b) holds.

Notice that if $X$ is a 1-complex properly imbedded in $M$, Claim 1.2 can be generalized to surfaces $S$ which are compressible in the complement of $X$; if $S$ is punctured by $X$, we can simply think of $S$ as being properly imbedded in the manifold $M-n(X)$.

\section{Proof of Lemma 2}

Notation: Since all of the surfaces under consideration are punctured 2-spheres, we will sometimes use incompressible and weakly incompressible in place of incompressible in the complement of the 1-skeleton and weakly incompressible in the complement of the 1-skeleton.

Lemma 2. Let $M_{0}$ be a component of $M-\Sigma$ with more than one boundary component. Then $M_{0}$ is a punctured 3-ball.

Proof. Let $T_{0}$ be the remnants of the 1-skeleton of $T$ in $M_{0} . T_{0}$ consists of imbedded arcs.

We need: 


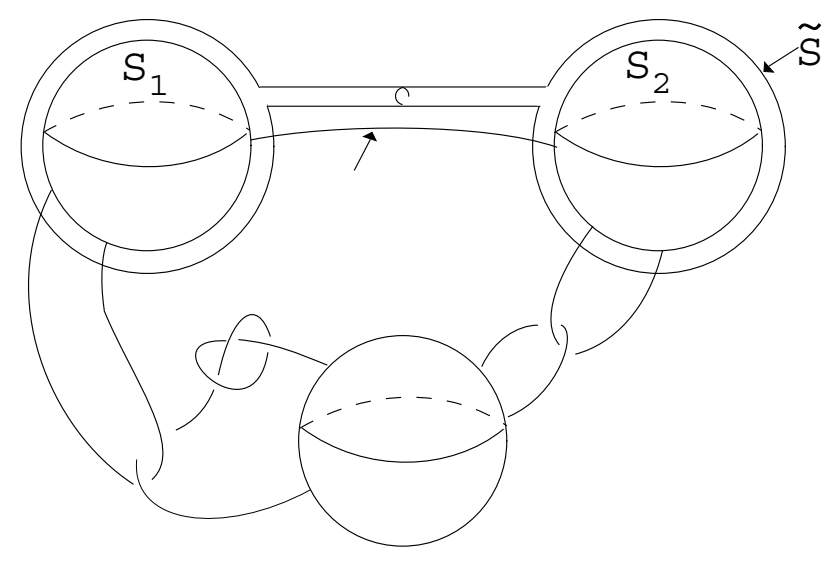

FIGURE 5

Subclaim 2.0.1. There exists an arc $\alpha$ of $T_{0}$ connecting distinct boundary components $S_{1}$ and $S_{2}$ of boundary $\left(M_{0}\right)$.

Proof. Suppose not. Then $M_{0}-n\left(T_{0}\right)$ is a connected manifold with more than one boundary component. Notice that the faces of the tetrahedra cut $M_{0}-n\left(T_{0}\right)$ into 3 -balls. But cutting a connected manifold with more than one boundary component open along disks yields a collection of components, at least one of which has more than one boundary component; in particular, it is not a collection of 3-balls. Hence such an $\alpha$ exists.

Construct a new 2-sphere $\tilde{S}$ by tubing push-offs of $S_{1}$ and $S_{2}$ together via a tube parallel to $\alpha$ (see figure 5 ).

$\tilde{S}$ is compressible to both sides in the complement of $T_{0}$; a meridian disk $\mathbf{E}$ of the tube is the unique compressing disk to one side (the inside), while a disk enclosing the arc $\alpha$ is a compressing disk to the outside. Notice that $\tilde{S}$ is weakly incompressible in the complement of $T_{0}$, since the boundary of every compressing disk on the side containing $\alpha$ must run over the tube. Let $\mathbf{D}$ be a maximal collection of disjoint compressing disks for $\tilde{S}$ in $M_{0}$ in the complement of $T_{0}$ on the outside. Let $\tilde{M}$ be the manifold obtained by attaching 2-handles to a small neighborhood of $\tilde{S}$ along $\partial \mathbf{D} \cup \partial \mathbf{E}$. Notice that $\tilde{M}$ is homeomorphic to a punctured 3-ball. We aim to show that $\tilde{M}$ is (more-or-less) all of $M_{0}$.

Claim 2.1. $\tilde{M}$ is homeomorphic to $M_{0}-\left(3\right.$-balls), hence $M_{0}$ is a punctured 3-ball.

Proof. The boundary of $\tilde{M}$ consists of a collection of punctured 2-spheres. The two inside $\tilde{S}, S_{1}^{\prime}$ and $S_{2}^{\prime}$, are parallel to $S_{1}$ and $S_{2}$. Let $\mathbf{S}=\partial \tilde{S}-\left(S_{1}^{\prime} \cup\right.$ 
$\left.S_{2}^{\prime}\right)$. Apply Claim 1.2 to $\tilde{S}$. Options 1,2 and 3 of Lemma 1.2 cannot occur, so $\partial \tilde{M}$ consists of a collection of punctured 2-spheres incompressible in the complement of $T_{0}$. By Claim 1.1, we can isotop $\tilde{M}$ in $M_{0}$ such that after the isotopy, $\partial \tilde{M}$ consists of a collection of normal 2-spheres and parallel 2 -spheres. We will continue to call the components of $\partial \tilde{M} S_{1}^{\prime}, S_{2}^{\prime}$ and $\mathbf{S}=\partial \tilde{S}-\left(S_{1}^{\prime} \cup S_{2}^{\prime}\right)$. Each component of $\partial \tilde{M}$ is a separating sphereotherwise we've just found a non-separating normal 2-sphere, which could not have been in our maximal collection $\Sigma$, a contradiction. $S_{1}^{\prime}$ and $S_{2}^{\prime}$ will remain parallel to $S_{1}$ and $S_{2}$; we can imagine pushing them out to $S_{1}$ and $S_{2}$. Let $S^{\prime} \subset \mathbf{S}$. If $S^{\prime}$ is a parallel 2-sphere, it must be parallel to the outside (the side disjoint from $\tilde{S})$, and we can fill in the $(3$-ball + arc) that it bounds. If $S^{\prime}$ is a normal 2-sphere, it must be parallel to some component of $\partial M_{0}$, since $\Sigma$ is maximal. It cannot be parallel to a boundary component of $M_{0}$ on the inside, since it contains at least two components $\left(S_{1}\right.$ and $\left.S_{2}\right)$ of $\partial M_{0}$ on the inside. Hence it must be parallel to a component of $\partial M_{0}$ on the outside, i.e., we can push it out to $\partial M_{0}$ disjoint from the rest of $\tilde{M}$. Hence each component $\partial \tilde{M}$ in $S$ can either be filled in with a 3 -ball or pushed out to the boundary of $M_{0}$. Conversely, each component of $\partial M_{0}$ will have one component of $\partial \tilde{M}$ pushed onto it by this process. Hence $\tilde{M}$ is homeomorphic to $M_{0}-$ (3-balls).

The claim concludes the proof of Lemma 2.

\section{An Unknotting Lemma}

This variant on the light bulb trick is a key ingredient in the proof of Lemma 4. It implies that if you attach a circular (and very flexible!) light bulb to the ceiling via a knotted cord you can unknot the cord without detaching it from the ceiling or the bulb, if you're willing to distort the bulb. Generalizations and further details of this lemma can be found in [5].

\subsection{The Fluorescent Light Bulb Trick.}

Lemma 3. Let $K$ be a closed connected subset of $S^{3}$, and let $v$ be a point in $S^{3}-K$. Let $\alpha, \beta$ be arcs connecting $v$ to a point $p$ on $\partial K$, coincident near $p$, with interiors disjoint from $K$. Then there is a homeomorphism $h$ of $S^{3}$, isotopic to the identity rel $v$, such that $h(K)=K, h(\alpha)=\beta$.

Proof. There is a homeomorphism $h_{\alpha}\left(h_{\beta}\right)$ of $S^{3}$ rel $v$ which shrinks $\alpha(\beta)$ to a very short arc, and straightens it out. It is helpful to imagine here that $\alpha(\beta)$ is made of very stiff wire threaded through a bead at $v: h_{\alpha}\left(h_{\beta}\right)$ first pulls $\alpha(\beta)$ almost completely through $v$, then straightens out the remaining short piece. Call this resulting straight $\operatorname{arc} \delta_{\alpha(\beta)}$. A rotation $r$ will take $\delta_{\alpha}$ to $\delta_{\beta}$. Then $h=\left(h_{\beta}^{-1}\left(r\left(h_{\alpha}\right)\right)\right)$ is the desired homeomorphism. 


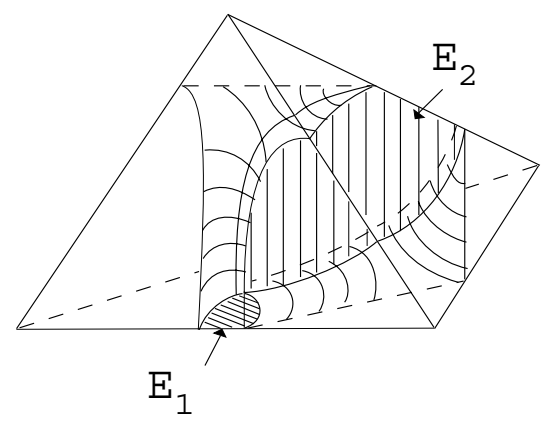

FIGURE 6

Corollary 3.1. Let $K$ be a closed connected subset of $B^{3}$ disjoint from $\partial B^{3}$, and let $v$ be a point on $\partial B^{3}$. Let $\alpha, \beta$ be arcs connecting $v$ to a point $p$ on $\partial K$, coincident near $p$, with interiors disjoint from $K$. Then there is a homeomorphism of $B^{3}$, isotopic to the identity rel $v$, such that $h(K)=K, h(\alpha)=\beta$.

Proof. Map $B^{3}$ into $S^{3}$ so that $\partial B^{3}$ maps to a point and use Lemma 3.

\section{Proof of Lemma 4}

Lemma 4. A component $M_{0}$ of type 3 is a 3-ball if and only if $M_{0}$ contains an almost normal 2-sphere.

Proof.

Step 1: We first show that if $M_{0}$ contains an almost normal 2-sphere then it is a 3 -ball.

Proof of step 1: Let $T_{0}$ be the remnants of the 1-skeleton of $T$ in $M_{0}$. Let $S$ be an almost normal 2-sphere in $M_{0}$. Examination of the single octagonal component of $S$ yields the information that $S$ is compressible to both sides in the complement of $T_{0}$, since one can double the obvious boundary compressions (see figure 6 ).

We need to show that $S$ is weakly incompressible in the complement of $T_{0}$. $S$ divides $M_{0}$ into two components, $M_{1}$ and $M_{2}$, with $\partial M_{0}=M_{1}$. Let $E_{1} \subset M_{1}$ and $E_{2} \subset M_{2}$ be the boundary compressing disks for $S$ arising from its octagonal component. Let $D_{1} \subset M_{1}$ and $D_{2} \subset M_{2}$ be compressing disks for $S$ in the complement of $T_{0}$. Suppose $\partial D_{1} \cap \partial D_{2}=\emptyset$. After an isotopy which maintains $\partial D_{1} \cap \partial D_{2}=\emptyset$, we can assume that $D_{1} \cup D_{2}$ intersects the 2-skeleton only in arcs. Minimize, up to isotopy maintaining $\partial D_{1} \cap \partial D_{2}=\emptyset$, the number of points in $\left(D_{1} \cup D_{2}\right) \cap(S \cap 2$-skeleton). Consider an outermost arc $\alpha$ in $D_{1}$ of $D_{1} \cap$ (2-skeleton). $\alpha$ cuts off a subdisk $D_{1}^{\prime}$ of $D_{1}$. $D_{1}^{\prime}$ lies entirely in some tetrahedron $H$. If $D^{\prime}$ lies in a tetrahedron not containing the octagonal component, we could reduce the 
number of components in $\left(D_{1} \cup D_{2}\right) \cap(S \cup 2$-skeleton $)$, maintaining $\partial D_{1} \cap$ $\partial D_{2}=\emptyset$, contradicting our hypothesis. Hence $D_{1}^{\prime}$ lies in the tetrahedron containing the octagonal component of $S$, and in fact $D_{1}^{\prime}$ must look like a push-off of $E_{1}$, disjoint from the edge incident to $E_{1}$. Applying the same argument to $D_{2}$ yields a contradiction, since $D_{1}^{\prime}$ and $D_{2}^{\prime}$ must intersect. Hence $S$ is weakly incompressible in the complement of $T_{0}$.

Let $\mathbf{D} \subset M_{1}$ and $\mathbf{E} \subset M_{2}$ be minimal complete collections of compressing disks for $S$ in the complement of $T_{0}$. The proof now is similar to the proof of Lemma 2 . Let $\tilde{M}$ be the manifold obtained by attaching 2-handles to a small neighborhood of $S$ along $\partial \mathbf{D} \cup \partial \mathbf{E}$. Notice that $\tilde{M}$ is homeomorphic to a punctured 3-ball. We aim to show that $\tilde{M}$ is (more-or-less) all of $M_{0}$.

Apply Claim 1.2 and Claim 1.1 to conclude, after an isotopy, that every component of $\partial \tilde{M}$ is either a parallel 2-sphere or a (separating) normal 2 -sphere. Since $\Sigma$ is maximal, any normal 2 -sphere in $M_{0}$ is parallel to $\partial M_{0}$. There is exactly one component, $S^{\prime}$, of $\partial \tilde{M}$, that separates $S$ from $\partial M_{0}$. Let $S^{\prime \prime}$ be any other component of $\partial \tilde{M}$. If $S^{\prime \prime}$ is normal, then it is boundary parallel, hence we have the almost normal 2-sphere $S$ lying between two parallel normal 2-spheres $\partial M_{0}$ and $S^{\prime \prime}$, a contradiction. So $S^{\prime \prime}$ must be a parallel sphere, and we can fill it in with a (3-ball+arc). Filling in all such $S^{\prime \prime}$ 's, we are left with the single component $S^{\prime}$ of $\partial \tilde{M}$, which cannot be a parallel 2-sphere, hence must be parallel to $\partial M_{0}$. Pushing this component out to $\partial M_{0}$ completes the argument.

Step 2: We now show that if $M_{0}$ is a 3-ball then $M_{0}$ contains an almost normal 2-sphere.

Proof of step 2: We adjust the definition of thin position for knots in the 3 -sphere [2]: assume that $T_{0}$ is a collection of arcs properly imbedded in the 3-ball $M_{0}$. Let $F$ be a foliation of $M_{0^{-}}$(point) with 2 -spheres, such that all but a finite number of the 2 -spheres intersect $T_{0}$ transversely, and every 2 -sphere in $F$ has at most one point of tangency with $T_{0}$. Call the 2spheres having a point of tangency with $T_{0}$ singular spheres, and all other 2 -spheres in $F$ transverse spheres. Between each adjacent pair of singular spheres, choose a transverse sphere $S_{0}$. Define the width of $T_{0}$ with respect to $F$ to be the sum over $i$ of [the number of times $T_{0}$ intersects $S_{i}$ ]. Define the width of $T_{0}$ to be the minimum width of $T_{0}$ with respect to $F$ over all possible foliations $F$. If the foliation $F$ realizes the width of $T_{0}$ then $T_{0}$ $($ rel $F)$ is in thin position.

Let $F$ be the foliation realizing the width of $T_{0}$. Beginning at $\partial M_{0}$, the foliation passes through critical levels with respect to $T_{0}$. We see a sequence of maxima with respect to $F$, then a sequence of minima, and so on. Call a transverse 2 -sphere in the region where the sequence shifts 
from maxima to minima (minima to maxima) a thick (thin) 2-sphere.

Call a simple closed curve lying in the interior of a face of a tetrahedron a simple curve.

Let $S$ be any transverse 2 -sphere in $F$. Suppose $S$ intersects the boundary of each tetrahedron in normal curves and simple curves.

Claim 4.1. Let $H$ be any tetrahedron in the triangulation of $M$. Then $S \cap \partial(H)$ contains no parallel curves of length greater than or equal to eight.

Claim 4.2. Let $H$ be any tetrahedron in the triangulation of $M$. Then $S \cap \partial(H)$ contains no curve of length greater than eight.

Claim 4.3. Let $H_{1}$ and $H_{2}$ be distinct tetrahedra in the triangulation of $M$. Then $S \cap \partial\left(H_{1}\right)$ and $S \cap \partial\left(H_{2}\right)$ do not both contain curves of length eight.

The proofs of Claims 4.1-4.3 proceed by exhibiting various violations of thin position.

Recall from Section 1 the following facts about normal curves on the boundary of a tetrahedron $H$ :

(1) A normal curve of odd length has length 3.

(2) There are no normal curves of length 6 .

(3) Any normal curve of length greater than 8 crosses some edge of $H$ at least 3 times.

For each tetrahedron $H, S \cap H$ is almost determined by $S \cap \partial H$; we can picture $S \cap H$ as a collection of disks $\mathbf{D}$ with $\partial \mathbf{D}=$ the normal curves of $S \cap \partial H$, connected together and to the faces by tubes, which may, of course, be quite complicated, and may run through each other.

$S$ divides $M_{0}$ into two pieces, the exterior, which contains $\partial M_{0}$, and the interior. Anything in the exterior of $S$ lies above $S$, anything in the interior lies below.

An upper (lower) disk for $S$ is a disk $K$ such that $\partial K=\alpha \cup \beta$, where $\alpha$ is an arc imbedded in $S, \partial \alpha=\partial \beta, \beta$ is a subarc of $T_{0}, K-\alpha$ intersects $S$ transversely, and a small product neighborhood of $\alpha$ lies above (below) $S$. Call $K$ a strict upper (lower) disk if $\beta$ lies entirely above (below) $S$. By an innermost disk argument, one can see that such a disk $K$ can be chosen to lie completely in the interior of $M_{0} . K$ describes an isotopy of $T_{0}$ in which the $\operatorname{arc} \beta$ can be replaced by the $\operatorname{arc} \alpha$, and then pushed slightly below (above) $S$. See [9] for further details.

Proof of claim 4.1. Suppose $S \cap \partial H$ contains parallel normal curves $c_{1}$ and $c_{2}$ of length greater than or equal to eight. Choose $c_{1}$ and $c_{2}$ to be adjacent in $\partial \mathrm{H}$, and such that $c_{2}$ bounds a disk $\mathrm{J}$ in $\partial H$ containing only normal 


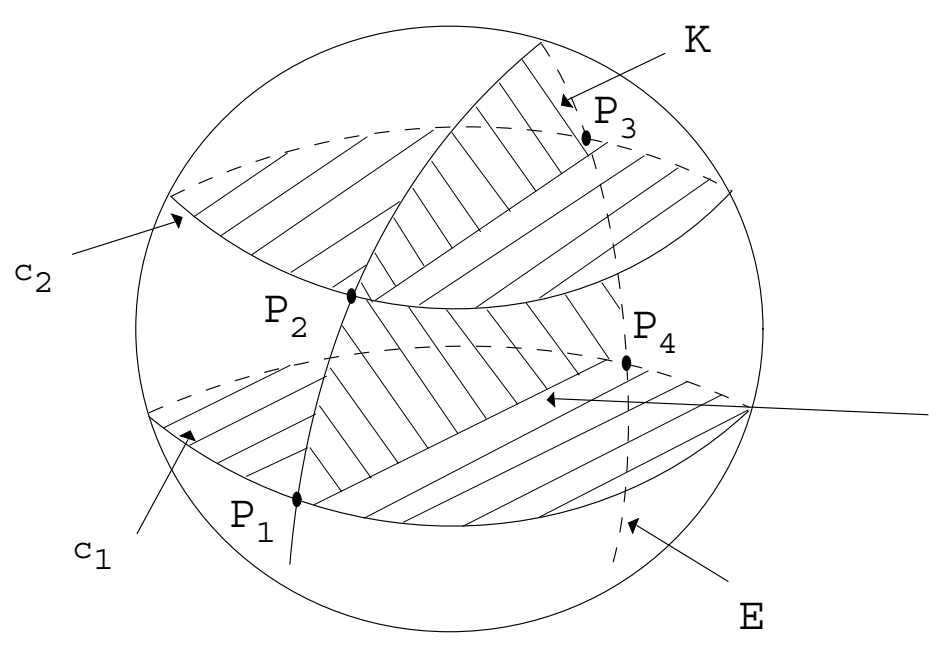

FIGURE 7

curves of length three. Let $E$ be one of the edges crossed at least twice by $c_{1}$ and $c_{2}$. Let $D_{1}$ and $D_{2}$ be disks in $\mathbf{D}$ with $\partial \mathrm{D}_{1}=\mathrm{c}_{1}, \partial D_{2}=\mathrm{c}_{2}$. Let $p_{1}, p_{2}, p_{3}, p_{4}$ be consecutive (on $E$ ) points of intersection between $E$ and $c_{1} \cup c_{2}$, where the segment of $E$ between $p_{2}$ and $p_{3}$ lies in J. So $p_{1}$ and $p_{4}$ are points on $c_{1}$, while $p_{2}$ and $p_{3}$ are points on $c_{2}$. Let $K$ be an imbedded disk in $H$, with $\partial K$ consisting of the piece $e$ of $E$ connecting $p_{1}$ to $p_{4}$, containing $p_{2}$ and $p_{3}$, together with an imbedded arc $\alpha$ in $D_{1}$ connecting $p_{1}$ to $p_{4}$. Further, choose $K$ so that $K$ intersects $D_{2}$ in a single imbedded arc connecting $p_{2}$ to $p_{3}$ (see figure 7 ).

$K$ acts as a simultaneous upper and lower disk for $T_{0}$; we now replace $e$ with $\alpha$ via an isotopy across $K$, which takes place in $M_{0}$, reducing the width of $T_{0}$, a contradiction. Hence $S \cap \partial H$ contains no parallel normal curves $c_{1}$ and $c_{2}$ of length greater than or equal to eight.

Proof of claim 4.2. Suppose $S \cap \partial H$ contains a normal curve $c$ with length greater than eight. Let $E$ be an edge of $H$ intersecting $c$ at least 3 times. Let $D \subset \mathbf{D}$ be the element of $\mathbf{D}$ with boundary $c$. Let $p_{1}, p_{2}$ and $p_{3}$ be consecutive points (on $E$ ) of intersection between $E$ and $c$ (see figure 8).

Then there exist upper and lower disks $K_{1}$ and $K_{2}$ for $S$ with respect to $T_{0}$ which intersect only at $p_{2}$. The boundary of $K_{1}$ consists of the piece of $E$ between $p_{1}$ and $p_{2}$, together with an arc $\alpha_{1}$ in $D$ connecting $p_{1}$ to $p_{2}$. The boundary of $K_{2}$ consists of the piece of $E$ between $p_{2}$ and $p_{3}$, together with an arc $\alpha_{2}$ in $D$ connecting $p_{1}$ to $p_{2}$, with $\alpha_{1} \cap \alpha_{2}=p_{2}$. The idea is to replace the piece of $E$ connecting $p_{1}$ to $p_{2}$ with $\alpha_{1}$ and the piece connecting $p_{2}$ to $p_{3}$ with $\alpha_{2}$. This can be done by an isotopy of $E$; 


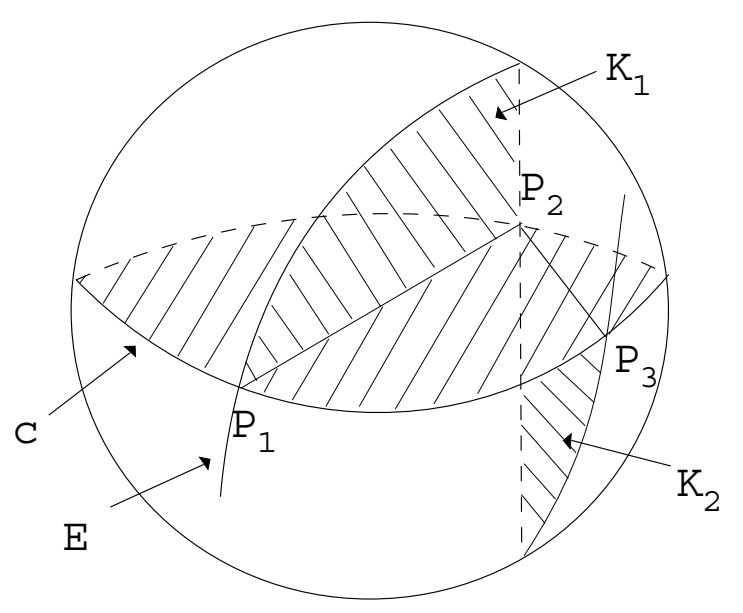

Figure 8

note that the isotopy in fact occurs entirely inside $M_{0}$, since no normal 2sphere disjoint from $S$ can intersect $K_{1}$ or $K_{2}$ non-trivially. This operation reduces the width of $T_{0}$, a contradiction. Hence $S \cap \partial H$ contains no normal curve $c$ with length greater than eight.

Proof of claim 4.3. Suppose $S \cap \partial\left(H_{1}\right)$ and $S \cap \partial\left(H_{2}\right)$ contain curves $c_{1}$ and $c_{2}$ of length eight. Let $D_{1}$ and $D_{2}$ be disks in $\mathbf{D}_{1}$ and $\mathbf{D}_{2}$ respectively with $c_{i}=\partial D_{i} . c_{1}$ crosses two edges of $H_{1}$ exactly twice. Let $e_{1 u}$ and $e_{1 l}$ be the segments of these edges disjoint from the vertices of $H$. Note that $e_{1 u}$ begins above $S, e_{1 l}$ begins below. Let $K_{1 u}$ be a disk imbedded in $H$ with boundary consisting of $e_{1 u}$ together with an $\operatorname{arc} \alpha_{1 u}$ in $D_{1}$ connecting the ends of $e_{1 u}$. Define $K_{1 l}$ similarly. We choose $\alpha_{1 u}$ and $\alpha_{1 l}$ to intersect in a single point. Define $e_{2 u}, e_{2 l}, K_{2 u}, K_{2 l}, \alpha_{2 u}$ and $\alpha_{2 l}$ similarly in $H_{2}$. We use $K_{1 u}$ and $K_{2 l}$ to reduce the width of $T_{0}$. If $e_{1 u}$ and $e_{2 l}$ are disjoint or intersect in a single (end)point, replace $e_{1 u}$ with $\alpha_{1 u}$ and $e_{2 l}$ with $\alpha_{2 l}$ via an isotopy. This reduces the width of $T_{0}$. The other possibility is that $e_{1 u}$, say, is a proper subarc of $e_{2 l}$. Then replacing $e_{2 l}$ with $\alpha_{2 l}$ reduces the width of $\mathrm{T}_{0}$, a contradiction. Hence $S \cap \partial\left(H_{1}\right)$ and $S \cap \partial\left(H_{2}\right)$ cannot both contain curves of length eight.

Claims 4.1-4.3 imply that if a transverse $S$ intersects the 2-skeleton only in normal and simple curves, then $S \cap$ (2-skeleton) consists of normal curves of lengths 3 and 4, simple curves and possibly one normal curve of length 8 .

Claim 4.4. There exists a transverse 2-sphere in the first thick region of F which intersects the 2-skeleton entirely in normal arcs and simple curves. 
Proof. This is a straightforward application of [2], Lemma 4.4. The idea is as follows: we can assume a transverse 2-sphere $S^{\prime}$ at the top of the thick region, i.e. immediately below a maximum of $T_{0}$, cuts off a boundary compressing disk, lying above $S^{\prime}$, in a face of one of the tetrahedra. This is an upper disk for $S^{\prime}$. Similarly a transverse 2-sphere $S^{\prime \prime}$ immediately above the minimum occurring at the bottom of the thick region cuts off a boundary compressing disk in a face which lies below $S^{\prime \prime}$, forming a lower disk for $S^{\prime \prime}$. Because $T_{0}$ is in thin position, we never see upper and lower disks for a transverse $S$ which are disjoint or which intersect at a single point in $S \cap T_{0}$; hence somewhere between $S^{\prime}$ and $S^{\prime \prime}$ we can find an $S$ with no upper or lower disks contained in the faces of the tetrahedra. This $S$ intersects the 2-skeleton in normal arcs and simple curves.

By Claims 4.1-4.3, this sphere $S$ intersects the boundary of each tetrahedron in simple curves, normal curves of length three and four, and possibly, on one tetrahedron, a single curve of length eight.

If $S \cap \partial H$ indeed contains a curve of length eight for some $H$, we are done; simply compress $S$ as much as possible (to both sides, in several steps) along simple curves and disks interior to the tetrahedra. The resulting collection of 2-spheres will all be normal, with the exception of the component containing the curve of length eight. This will be the desired almost normal 2-sphere.

Claim 4.5. $S \cap \partial H$ contains a normal curve of length eight for some $H$.

Proof. Suppose not. Then compressing $S$ as much as possible, as described above, yields a collection of normal 2-spheres. Since the only normal 2sphere in $M_{0}$ is $\partial M_{0}, S$ is $n$ copies of $\partial M_{0}$ connected by tubes. The tubes may run through each other. We aim to show that this is impossible.

Case 1: Suppose $S$ consists of two copies of $\partial M_{0}$ tubed together. Recall, however, that $S$ is boundary compressible above. Let $D$ be a meridian disk of the single tube. $D$ lies above $S$. By an outermost arc argument, there exists a boundary compressing disk for $S$ disjoint from $D$. But then there exists a boundary compressing disk for one of the two copies of $\partial M_{0}$, a contradiction.

Case 2: $S$ consists of $n>2$ (nested) copies of $\partial M_{0}$ connected by tubes. Beginning with the outermost copy of $\partial M_{0}$, we can number the copies of $\partial M_{0}$. Let $R$ be the third copy of $\partial M_{0}$. Let $P$ be the piece of $R$ which is coincident with $S . P$ is a connected planar surface (see figure 9 ). We will construct a new and thinner foliation on $M_{0}$.

Since $R$ is parallel to $\partial M_{0}$, all the arcs exterior to $R$ are parallel, say to a single arc $\alpha$, and connect $R$ to $\partial M_{0}$. Imagine that all the arcs exterior to $R$ are contained in a thick copy of the $\operatorname{arc} \alpha$. Since $P$ is a subsurface 


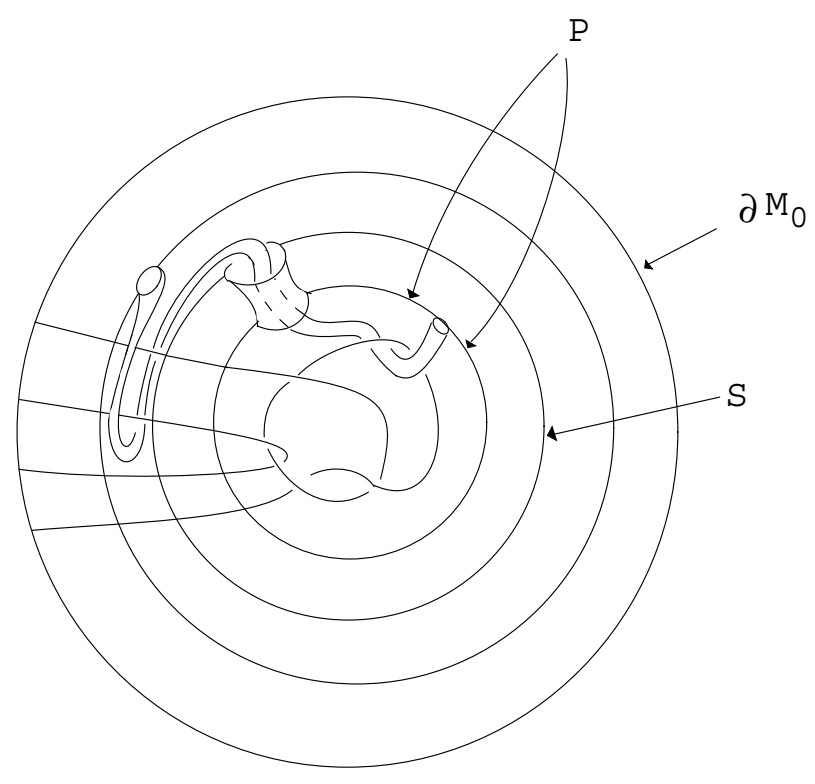

FiguRE 9

of the level sphere $S$, we can connect $\partial M_{0}$ to $P \cap \alpha$ via an arc $\beta$ which intersects each 2-sphere in the foliation at most once. Now let $K=n(P \cup$ $(\operatorname{arcs}$ interior to $R)$ ), and apply Corollary 3.1 to replace $\alpha$ with $\beta$ via a homeomorphism $h$ of $M_{0}$. The original foliation $F$ now describes a new foliation $\tilde{F}$ with respect to $h\left(T_{0}\right)$ in $M_{0}$ - (point), with the properties:

(1) width(arcs interior to $R$ with respect to $F)=$ width(arcs interior to $R$ with respect to $\tilde{F}$.)

(2) The arcs exterior to $R$ contribute nothing to the width of $h\left(T_{0}\right)$.

Since any arc exterior to $R$ contributed at least two to the width of $T_{0}$ with respect to $F, h\left(T_{0}\right)$ is thinner with respect to the foliation $\tilde{F}$.

This completes the proof of Lemma 4.

\section{Finding almost normal 2-spheres}

Fix a tetrahedron $H$ in $M$, and fix a normal curve $c$ of length eight on the boundary of $H$ (there are three choices for $c$ ). We modify standard normal surface theory algorithms to search for an almost normal 2-sphere $S$ with octagonal component bounded by $c$.

Let $\mathcal{Z}$ be a finite system of linear equations such that a positive integral solution to $\mathcal{Z}$ corresponds to a (possibly immersed) surface $F$, where, except in $H, F$ is composed of normal triangles and quadrilaterals. In $H, F$ is 
composed of normal triangles and octagonal components with boundaries parallel to $c$. Just as for normal surfaces, there exists a finite collection $F_{1}, F_{2}, \ldots, F_{n}$ of fundamental solutions to $\mathcal{Z}$. Any solution to $\mathcal{Z}$ can be written as a finite sum of fundamental solutions, where the addition operation is a regular alteration (a double-curve sum, where the the direction of the sum is chosen to ensure the resulting surface will be normal) along curves of intersection. We would like to show that if $M$ contains an almost normal 2-sphere, then one of the $F_{i}$ 's is an almost normal 2-sphere. Since we can find the $F_{i}$ 's algorithmically (see [4],[6]), this would finish the problem, provided we repeat the search procedure for each normal curve of length eight in each tetrahedron.

Unfortunately this doesn't quite work; we will use in addition the observation that we need only search for almost normal 2-spheres lying in $M_{0}$.

Lemma 5. If there exists an almost normal 2-sphere $S$ in $M_{0}$ then there exists one which is a fundamental solution to $\mathcal{Z}$.

Proof. We note :

(1) Recall that $H_{1}\left(M ; \mathbb{Z}_{2}\right)$ is trivial, hence $M$ contains no closed nonorientable surfaces.

(2) Euler characteristic is additive under the addition operation.

Let $S$ be an almost normal 2-sphere in $M_{0}$. If $S$ is not a fundamental solution to $\mathcal{Z}$, then it can be written as the sum of fundamental solutions, so $S=G_{1}+G_{2}+\cdots+G_{k}$, where each $G_{i}$ is a fundamental solution to $\mathcal{Z}$. One of the $G_{i}$ 's is an almost normal surface and the rest are normal surfaces. Since $S$ lies in $M_{0}$, each of the $G_{i}$ 's also lies in $M_{0}$. By notes 1 and 2 , at least one of the $G_{i}$ 's, say $G_{1}$, is a 2 -sphere. If it is an almost normal 2-sphere, we are done. If not, it is a normal 2-sphere, hence parallel to $\partial M_{0}$.

There exists an isotopy $i$ of $M_{0}$, preserving the normal structure or almost normal structure of the $G_{i}$ 's, such that $i\left(G_{1}\right)$ is disjoint from $i\left(G_{2} \cup\right.$ $\left.\cdots \cup G_{k}\right)$. To see the isotopy, in each tetrahedron, simply push the pieces of $G_{2} \cup \cdots \cup G_{k}$ into $M_{0}$ disjoint from the $(2$-sphere $) \times I$ bounded by $G_{1}$ and $\partial M_{0}$.

Since $i$ doesn't change the normal or almost normal structure of $G_{1}$ or $G_{2} \cup \cdots \cup G_{k}$,

$$
G_{1}+G_{2}+\cdots+G_{k}=i\left(G_{1}\right)+i\left(G_{2}\right)+\cdots+i\left(G_{k}\right) .
$$

But $i\left(G_{1}\right)+i\left(G_{2}\right)+\cdots+i\left(G_{k}\right)$ is not a connected surface. Hence $S$ cannot be written as a non-trivial sum of solutions to $\mathcal{Z}$, hence $S$ must be a fundamental solution to $\mathcal{Z}$. 


\section{Acknowledgements}

I would like to thank Marty Scharlemann for helpful conversations, particularly on the proof of (the critical) Claim 4.5.

\section{References}

[1] A. Casson and C. McA. Gordon, Reducing Heegaard splittings, Topology and its Applications 27 (1987).

[2] D. Gabai, Foliations and the topology of 3-manifolds III, J. Diff. Geometry 26 (1987).

[3] W. Haken Theorie der Normalflachen, Acta. Math. 105 (1961), 245-375.

[4] G. Hemion The classification of knots and 3-dimensional spaces, Oxford University Press, 1992.

[5] J. Hass and A. Thompson Fluorescent light bulbs and the unknotting of arcs in manifolds, preprint.

[6] W. Jaco and U. Oertel, An algorithm to decide if a 3-manifold is a Haken manifold, Topology 23 (1984).

[7] W. Jaco and J. Tollefson, to appear.

[8] H. Rubinstein The solution to the recognition problem for $S^{3}$, lectures, Haifa, Israel, May 1992.

[9] M. Scharlemann and A. Thompson, Thin position and Heegaard splittings of the 3-sphere, preprint.

Department of Mathematics, University of California, Davis, CA 95616

E-mail address: thompson@math.ucdavis.edu 